\title{
Virulence factors of enteropathogenic Escherichia coli
}

\author{
D. LAW
}

Department of Microbiology, Freeman Hospital, Freeman Road, Newcastle upon Tyne, NE7 7DN

\section{Introduction}

Escherichia coli is part of the normal large-bowel flora of man and animals. Although most strains of $E$. coli are non-pathogenic in the intestine, some can produce diarrhoea and by a number of distinct mechanisms.

E. coli was first incriminated as an enteropathogen when Bray (1945) demonstrated that an antigenically distinct strain of $E$. coli was responsible for an outbreak of infantile diarrhoea. These strains were named enteropathogenic $E$. coli (EPEC) by Neter et al. (1955). Studies in many locations showed that EPEC strains were isolated with greater frequency from infants with diarrhoea than from healthy infants. Identification of EPEC strains isolated from faecal samples was by $\mathrm{O}$ serotype determination since virulence mechanisms could not be detected in these strains (Levine and Edelman, 1984).

In the 1970 s it was shown that some strains of $E$. coli produced a heat-labile (LT) or a heat-stable (ST) toxin or both, or had entero-invasive properties. These strains were classed enterotoxigenic (ETEC) and entero-invasive (EIEC) E. coli, respectively (Rowe, 1979). Examination of EPEC strains for the above described virulence traits yielded in the main negative results (Goldschmidt and DuPont, 1976; Gross et al., 1976; Levine et al., 1978). Some workers believed that the lack of identifiable virulence properties in EPEC strains justified the abandonment of their serotyping (Sack, 1976); others suggested that mechanisms distinct from those described might exist in EPEC strains and that serotyping should be continued (Rowe et al., 1975). This controversy was to some extent resolved when Levine et al. (1978) showed that EPEC strains negative in tests for LT, ST and invasiveness were, nevertheless, able to induce diarrhoea in human volunteers. This observation provoked intensive study of the virulence characteristics of $E$. coli and it is now apparent that strains can produce distinct intestinal disease in man by several different

Received 1 Sep. 1987; accepted 13 Oct. 1987. mechanisms. The main features of these strains of E. coli are shown in the table.

The function of this review is to describe in detail the virulence attributes of enteropathogenic strains of $E$. coli and their relationship with the virulence factors of other strains of $E$. coli and other enteric pathogens.

\section{Enterotoxins}

Animal studies. Early studies of enterotoxic activity in EPEC strains used the rabbit ileal-loop (RIL) model, a technique widely used for detecting enterotoxins and enterotoxigenic organisms that induce dilation of the ileal loop and cause fluid secretion into the lumen (De and Chaterjee, 1953). De et al. (1956) observed that three strains of EPEC could induce fluid accumulation and dilation of the RIL. Taylor et al. (1958) similarly detected enterotoxic activity in an EPEC strain of serogroup O26 and showed that ileal loops varied in their reactivity with the outcome being influenced by the strain of rabbit, diet and the bacterial growth medium.

Among EPEC strains of various serotypes isolated from children with and without diarrhoea, Taylor et al. (1961) showed that only strains isolated from symptomatic infants induced fluid secretion in the RIL. A labile material isolated from those strains was absent, however, from strains of similar antigenic structure isolated from other sources. This labile material was thought to be responsible for producing diarrhoea.

Sakazaki et al. (1974) examined 40 EPEC strains of which 27 caused dilation in the RIL. For any positive strain, however it was not uncommon to obtain only one of two or three loops positive. With some strains, culture filtrates gave better results than live cultures and vice versa. A rough variant of a strain able to produce dilation in the RIL was shown to be negative in this system. Culture filtrates of both smooth and rough strains could induce dilation suggesting a role for the $\mathrm{O}$ antigen in the production of fluid secretion by live organisms.

In contrast to the results described by Sakazaki 
Table. Properties of E. coli strains

\begin{tabular}{|c|c|c|}
\hline Category & Serogrouping & Virulence factors \\
\hline $\begin{array}{l}\text { Enterotoxigenic } E \text {. coli } \\
\quad \text { (ETEC) }\end{array}$ & $\begin{array}{l}\text { O6, O8, O15, O20, O25, O27, O63, O78, O80, } \\
\text { O85, O115, O128ac, O139, O148, O153, } \\
\text { O159, O167 }\end{array}$ & $\begin{array}{l}\text { Heat-labile (LT) and heat-stable (ST) toxins; } \\
\text { adhesive factors CFAI, CFAII and E } 8775\end{array}$ \\
\hline $\begin{array}{l}\text { Entero-invasive } E . \text { coli } \\
\text { (EIEC) }\end{array}$ & $\begin{array}{l}\text { O28ac, O29, O124, O136, O143, O144, O152, } \\
\text { O164, O167 }\end{array}$ & Epithelial invasion; Shiga toxin \\
\hline $\begin{array}{l}\text { Enterohaemorraghic } E \text {. coli } \\
\quad \text { (EHEC) }\end{array}$ & $\mathrm{O} 157, \mathrm{O} 26, \mathrm{O} 111$ & High levels of Shiga toxin; fimbrial adhesin \\
\hline $\begin{array}{l}\text { Entero-adherent } E \text {. coli } \\
\quad \text { (EAEC) }\end{array}$ & $?$ & Epithelial adherence \\
\hline $\begin{array}{l}\text { Enteropathogenic } E \text {. coli } \\
\text { (EPEC) }\end{array}$ & $\begin{array}{l}\text { O18ac, O26, O44, O55, O86, O111, O114, } \\
\text { O119, O125, O126, O127, O128, O142 }\end{array}$ & $\begin{array}{l}\text { Shiga toxin production; fimbrial or outer-mem- } \\
\text { brane protein adhesin in some strains }\end{array}$ \\
\hline
\end{tabular}

et al. (1974), Zinkernagel and Colombini (1975) found that various EPEC strains could cause fluid secretion but they were unable to detect enterotoxins in whole-cell lysates or culture supernates. It was suggested that poor production of toxin in vitro might account for this finding. A further study with the RIL model showed that only 3 of 48 EPEC strains were reactive (Goldschmidt and DuPont, 1976). Two of these were of serogroup O26, the other belonged to serogroup O111. Similar findings were obtained by Polotsky et al. (1977) who, with live cultures, showed that only two strains of serogroup $\mathrm{O} 26$ gave a constant response; some strains of serogroup O111 showed variable results, whereas strains of other serotypes gave negative results. Gorbach and Khurana (1972) found that an infant RIL was more sensitive than an adult RIL for detecting enterotoxic activity in EPEC strains and, with this model, demonstrated enterotoxic activity in EPEC strains of various serotypes.

Other workers, unable to obtain reactions with EPEC strains in the RIL model, obtained better results with infant mouse ileal loops, but again animal-to-animal variation was also found (Punyashthiti and Finkelstein, 1971). With the more sensitive rabbit-skin permeability-factor $(\mathrm{PF})$ test, in which toxins induce distinct skin lesions, Evans et al. (1973) detected enterotoxic activity in EPEC strains. The activity differed quantitatively and qualitatively from that of similar preparations from ETEC strains and again false-negative results were not uncommon even when concentrated culture supernates of EPEC strains were tested. Strains of proven pathogenicity have been shown to be nonenterotoxigenic in the PF test (Robins-Browne et al., 1982) and the infant RIL model, although reactivity was found in a canine-loop system (Levine et al., 1978).

Klipstein et al. (1978), using a perfused ratjejunum assay, showed that a number of EPEC strains were able to elaborate potent heat-labile and heat-stable enterotoxins which induced fluid secretion in this system. These toxins were distinct from LT and ST of ETEC strains and there was a correlation in this assay between the potency of the toxin and the severity of the disease caused by a particular strain of EPEC.

Although the results from these studies are often contradictory, they do suggest on balance that some form of enterotoxic product is elaborated by EPEC strains and that it is distinct from both LT and ST. The models which have been used, however, appear to lack sensitivity and show marked animal-toanimal variation, which may explain in part the lack of consistency of results. It is also likely that there is variation in the yields of toxin from different EPEC strains. Cultural conditions and different preparation methods may also affect the potency of enterotoxin preparations. It is apparent, however, that amongst EPEC strains, those belonging to serogroup $\mathrm{O} 26$ and, to a lesser extent, serogroup O111 produce higher yields of toxin, or are more likely to produce toxin, than strains of other serotypes.

Tissue-culture methods. Because the heat-labile toxin (LT) of E. coli and cholera toxin (CT) itself were found to produce distinct morphological changes on various tissue-culture cells, e.g., Chinese hamster ovary cells (CHO) (Guerrant et al., 1974), these methods were applied to EPEC strains. Numerous studies showed, however, that the majority of EPEC strains did not produce these 
toxins (Goldschmidt and Dupont, 1976; Gross et al., 1976; Levine et al., 1978) and Robins-Browne et al. (1982) using gene probes to detect nucleotide sequences for LT and ST, showed that EPEC strains did not have the genetic information to code for these toxins.

Adenylate cyclase-stimulating activity. The elucidation of the mode of action of $E$. coli $\mathrm{LT}$ and CT as stimulators of membrane adenylate cyclase (AC) prompted investigation of EPEC strains for toxins with a similar mode of action. Scheftel et al. (1980) detected a protein in culture supernates of EPEC strains that could stimulate the AC activity of ratheart membranes in a manner similar to CT. The $\mathrm{AC}$-stimulating factor was isolated from an EPEC strain of serogroup $\mathrm{O} 111$ and found to be a heatlabile protein the mol. wt of which was $30 \times 10^{3}$; some preparations, however, were complexed with polysaccharide and were heat-stable. The toxin stimulated AC and induced fluid secretion in the RIL though to a lesser extent than CT. A similar toxin isolated from an EPEC strain of serotype O126 stimulated AC activity in infant-rabbit intestinal tissue but not adult tissue (Kantor et al., 1974). Long-Krug et al. (1984) examined EPEC strains and showed that none produced an ACstimulating factor and Law et al. (1987), using strains known to be pathogenic in volunteers, showed that culturing these strains in conditions known to promote enterotoxin production by other pathogens did not lead to the detection of an ACstimulating factor in those strains.

The conflicting results from these studies may be due to differences in the assay conditions employed. The ATP concentration is particularly important, and AC-stimulating activity was detected only in the assays in which high concentrations of ATP were employed. CT and LT can bring about adenylate-cyclase stimulation when the ATP concentration is low, although some toxins, e.g., Shiga toxin, can activate $\mathrm{AC}$ when the ATP concentration is high (Charney et al., 1976). Such a toxin may be produced by EPEC strains but consensus of opinion is that EPEC strains do not elaborate an LT-like toxin.

\section{Cytotoxins}

As well as examining EPEC strains for toxins that can induce fluid secretion from the intestine, many workers have also examined these strains for cytotoxins, i.e., toxins that damage and kill cells.

Vero cytotoxin. In 1977, a cytotoxin shown to be produced by some strains of $E$. coli, notably of serogroup O26 (Konowalchuk et al., 1977), was detected by its cytotoxic effect on Vero tissueculture cells, was distinct from LT and was called Vero toxin (VT). It did not produce any change on $\mathrm{CHO}$ or $\mathrm{Y} 1$ adrenal cells and little response with RIL. A further study showed that VT had a mol. wt of $28 \times 10^{3}$, that it produced mild fluid secretion in the RIL but did not stimulate AC (Konowalchuk et al., 1978).

Examination of 252 strains of $E$. coli for VT found that only three strains gave positive results; these belonged to serotype O26, but another five strains of that serotype gave negative results. Removal of plasmids from a toxigenic strain did not affect toxin production (Wade et al., 1979) and Scotland et al. (1983b) found that VT genes were carried on bacteriophage in one of their strains. In another study of 253 EPEC strains from 11 different O-serogroups, only 25 produced VT and of these, 23 belonged to serotype O26 (Scotland et al., 1980). The same study suggested that although VT might have a role in the pathogenesis of EPEC diarrhoea, other factors were likely to be involved because some VT-negative strains were known to be pathogenic in human volunteers. Wade et al. (1979) also presented evidence of the possible significance of VT when they showed that all three VT-positive strains of serogroup $\mathrm{O} 26$ in their study were associated with bloody diarrhoea; the diarrhoea caused by EPEC strains is not usually bloody.

It seems likely that, in previous animal studies, the toxic activity detected in EPEC strains was due to VT because of the strong association of positive reactions in the RIL by strains of serogroup $\mathrm{O} 26$ that also produce VT.

In 1982 strains of $E$. coli belonging to serogroup O157, a type not previously recognised as pathogenic, were implicated in outbreaks of haemorrhagic colitis in the USA (Riley et al., 1983). Although these organisms were found to be noninvasive and produced neither LT nor ST, they elaborated large quantities of VT (Johnson et al., 1983; O'Brien et al., 1983); strains of E. coli of O157 were also implicated in cases of haemolytic-uraemic syndrome (Karmali et al., 1983b). It was proposed that haemorrhagic colitis and haemolytic-uraemic syndrome were different manifestations of the same deleterious effect of VT (Karmali et al., 1983a).

Strains of $E$. coli elaborating large amounts of VT and implicated as a cause of haemorrhagic colitis have been termed enterohaemorrhagic $E$. coli (EHEC) (Levine and Edelman, 1984) and Levine (1987) has suggested that EPEC strains of serogroup $\mathrm{O} 26$ be classed as EHEC because they resemble EHEC strains rather than EPEC strains in that they produce large quantities of VT and 
possess a similar plasmid of mol. wt $60 \times 10^{6}$ that encodes a distinct adhesin.

Shigella dysenteriae serotype 1-like toxin. O'Brien et al. (1982) observed that EPEC strains elaborated a toxin similar to the cytotoxin of Sh. dysenteriae serotype 1 (SDT) when grown in iron-depleted media. Some strains produced large quantities of this toxin, particularly those of serogroup $\mathrm{O} 26$. Other EPEC strains produced smaller amounts including strains previously used in feeding studies; non-pathogenic strains of $E$. coli elaborated trace amounts only of toxin. It was later shown that VT and SDT are almost identical (O'Brien et al., 1983) there being minor differences only between the EPEC toxin and that of Sh. dysenteriae (O'Brien and LaVeck., 1983). The heat-labile toxic product detected by Klipstein et al. (1978) has been shown to be neutralisable with antibody raised against Shiga toxin (Levine and Edelman, 1984).

To ascertain the importance of the toxin in EPEC strains, some large scale studies have been performed, in one of which $79 \%$ of EPEC strains produced SDT as compared with only $24 \%$ of strains of $E$. coli from healthy individuals (Cleary et al., 1985). Another study revealed that $59 \%$ of strains from outbreaks of diarrhoea were SDT producers. Some strains of $E$. coli, however, that gave negative results when cell sonicates were used, gave positive results when French-press lysates were tested (Marques et al., 1986). Whether all EPEC strains produce SDT to some extent, and whether the inability to detect it results from a lack of sensitivity in the assay system, remains to be determined.

A role has been ascribed to this toxin in strains of $E$. coli which produce large quantities of SDT from serogroups $\mathrm{O} 157$ and $\mathrm{O} 26$, in which it is believed to be responsible for the production of bloody diarrhoea and haemolytic-uraemic syndrome (Karmali et al., 1983a). The toxin is thought to be involved in EPEC disease but conclusive proof of this is lacking. The main doubts arise because of the very small quantities of toxin that are produced (O'Brien et al., 1982). Toxin production in vivo, however, may be greater and toxin delivery to the epithelium may be facilitated by the adhesive capabilities of the organism. Further doubt arises because SDT can be detected in nonpathogenic strains of $E$. coli (O'Brien et al., 1982; Cleary et al., 1985) and in bacteria possessing other potent enterotoxins, e.g., $V$. cholerae and ETEC strains (O'Brien et al., 1982; 1984a). The role of SDT in EPEC disease is most likely to be resolved by feeding studies with toxigenic and non-toxigenic derivatives of EPEC strains.
Several groups have shown that two antigenically distinct forms of cytotoxin from EPEC and EHEC strains exist, only one of which is neutralisable with antibody to Shiga toxin (Scotland et al., 1985; Strockbine et al., 1986). Both toxins probably share some common amino-acid sequences because some DNA relatedness has been demonstrated (Scotland et al., 1985). In addition, the toxin genes in both classes of organism have been shown to be carried by lysogenic phages (O'Brien et al., 1984b; Smith et al., 1984; Karch et al., 1987). This observation is of interest because genes for diphtheria toxin are also phage-mediated and production of that toxin is regulated by iron metabolism.

How SDT induces diarrhoea is as yet unclear, although cytotoxic and enterotoxic properties have been demonstrated (Keusch et al., 1981). Some workers report the inability of the toxin to stimulate adenylate cyclase (Flores et al., 1974; Donowitz et al., 1975), whereas others have detected AC stimulation in assays with high substrate (ATP) concentrations (Charney et al., 1976). Whether this is relevant in vivo has yet to be determined. In previous studies in which AC-stimulating activity was detected in EPEC strains, it is likely that this was caused by SDT, for high concentrations of ATP were used in those assays.

\section{EPEC adherence}

Adherence is an important factor in the initiation of many bacterial infections and in this connection $E$. coli is no exception. Human strains of ETEC produce fimbrial structures called colonisation factors, that bind to receptors on the intestinal epithelium and mediate adhesion. Although EPEC strains lack these structures, they have been investigated for the presence of other adhesins.

Type-1 fimbriae. Evans et al. (1979), examining the haemagglutination (HA) reactions of EPEC strains, showed that $42 \%$ of them had a distinct HA pattern termed HAIII that was very similar to the pattern given by common type 1-fimbriate bacteria, except that human red-blood cells were not agglutinated. The fimbriae-mediating HAIII are antigenically similar to type-1 fimbriae (Sherman et al., 1985). Type-1 fimbriae have been shown to mediate adherence to intestinal mucosa although strains expressing these fimbriae are variable in this ability. It has been suggested that these differences may be related to the hydrophobicity and charge of surface components of the bacterial cells (Sherman et al., 1985). Not all EPEC strains, however, carry type-1 fimbriae and other structures are likely, therefore, to be involved in the adhesion process. 
HEp2 adherence. Cravioto et al. (1979) demonstrated that $80 \%$ of EPEC strains showed mannoseresistant adherence to HEp2 tissue-culture cells whereas ETEC and normal-flora strains of E. coli rarely adhered. Non-adhesive EPEC strains were found more frequently among strains isolated from early outbreaks, a finding that was considered to be caused by loss of a plasmid-mediated adhesin. Scotland et al. (1983a) concluded that the adhesin was probably non-fimbrial in nature although its exact form was not known.

A plasmid of mol. wt $50-70 \times 10^{6}$, identified in 31 of 32 EPEC strains, was found to code for HEp2 adherence. Transfer of the plasmid, designated pMAR2, to $E$. coli strain K12 carried with it the ability to adhere to HEp2 cells. Deletion of pMAR2 from an EPEC strain, furthermore, resulted in loss of adherence to HEp2 cells and to the intestinal tissue of colostrum-deprived piglets (Baldini et al., 1983). The adhesin coded by this plasmid has been termed EPEC adhesive factor (EAF) (Levine et al., 1985).

Scaletsky et al. (1984) studied the adherence of EPEC strains to HeLa cells and noted that adhesion occurred in two distinct ways; localised adherence (LA) in which organisms attached to one or two small areas of the cell surface or diffuse adherence (DA) in which organisms attached to the whole of the cell surface. LA was associated with EPEC strains of serogroups O55, O86, O111, O119, O127, $\mathrm{O} 128$ and $\mathrm{O} 142$, the serogroups most commonly associated with diarrhoeal disease. EPEC strains belonging to these serogroups have been designated class-I EPEC, suggesting an important role for the plasmid-coded factor EAF. Class-II EPEC strains include some which adhere diffusely to HEp2 cells and others that do not adhere, and serogroups O44 and $\mathrm{O} 114$ are involved (Nataro et al., 1985a). The virulence of some class-II organisms has been clearly demonstrated in feeding studies (Levine and Edelman, 1984; Levine et al., 1985).

Various studies have addressed the nature of EAF, the binding of EAF to cellular receptors, and its role in virulence. The latter was convincingly demonstrated when an EPEC strain lacking the plasmid responsible for EAF was found to have considerably reduced virulence for human volunteers (Levine et al., 1985). In that same study it was established that an antibody response was mounted against an outer-membrane protein of mol. wt $94 \times$ $10^{3}$ encoded by the plasmid and possibly involved in the adhesion of EPEC strains that show LA. In a study of the LA adhesin (Andrade and Santa Rosa, 1986a) neither fimbriae nor capsules were demonstrable on an LA-positive strain. The adhesin was susceptible to trypsin treatment and was strongly associated with the cell, again suggesting the involvement of an outer-membrane protein in adhesion. Binding of LA-positive strains to HEp2 cells could be inhibited by carbohydrates although there was wide variation in the degree of inhibition by any particular carbohydrate even among strains of EPEC belonging to the same O-serogroup; that suggests structural variations of an adhesin which binds to glycoprotein receptors on the cell surface.

The nature of the EAF receptor has not yet been determined but some studies have provided important information on this subject. Froman et al. (1984) revealed that some EPEC strains were able to bind fibronectin, a glycoprotein present on the surface of many cells. Characterisation of one strain established the existence of two classes of bacterial receptor for fibronectin and showed that these differed in their affinity for fibronectin and susceptibility to binding-inhibition by other glycoproteins.

Working with pairs of EPEC strains with and without the pMAR2 plasmid, Wadstrom et al. (1986) showed that the plasmid-containing strain bound fibronectin to a greater degree than a plasmid-free strain, suggesting that fibronectin may be a receptor for the EAF of EPEC strains. However, because some fibronectin is bound by strains lacking EAF, the demonstration of two distinct receptors in EPEC strains and the variations in adhesion-inhibition by different carbohydrates suggest that other adhesins or receptors may be involved.

A DNA probe has been developed that detects the $p$ MAR-coded EAF and can be used for the direct detection of EPEC strains in faecal samples. The use of DNA probes has shown that the genes encoding LA and DA are distinct (Nataro et al., 1985b). There are, nevertheless, discrepant findings. Thus, in one study it was reported that all five EPEC strains of serogroup O86 were LA-positive (Scaletsky et al., 1984) whereas in another study none of 19 strains was LA-positive (Nataro et al., $1985 b$ ). It is possible that the possession of different adhesins by EPEC strains may vary geographically.

The DA characteristic is likely to be important among EPEC strains which have this property but further work is needed to characterise this adhesin. There is evidence that the adhesin confers hydrophobic properties on DA-positive strains that may assist in the adhesion process (Nataro et al., 1985a).

EPEC adherence in vivo. As well as studying EPEC adherence by in-vitro systems, adhesion in vivo has been examined in studies that used animal models and, more importantly, adhesion to human tissue during actual infections. 
Ultrastructural studies of EPEC infection in vivo were first performed on gnotobiotic piglets by Staley et al. (1969) who demonstrated distinct lesions in which lengthening and disruption of the microvilli and intimate attachment of bacteria to the epithelial cell surface of the intestine occurred. Similar histological findings have also been noted in rabbit intestinal tissue by Polotsky et al. (1977) and in colostrum-deprived piglets by Moon et al. (1983).

In a study of human infection, biopsies were taken of duodenal and colonic mucosa from an infant with protracted diarrhoea caused by EPEC strains of serogroup O125ac. Examination of the biopsies revealed lesions identical to those already described (Ulshen and Rollo, 1980). Rothbaum et al. (1982) investigated an outbreak of chronic protracted diarrhoea caused by EPEC of serogroup O119. Biopsies of jejunal and rectal mucosa again revealed findings similar to those already described. It was also noted that where bacteria adhered to the mucosa, the epithelial membrane protruded so that the bacteria appeared to rest on a pedestal. These strains were found to produce fimbriae associated with HAIII pattern and a capsule. The role of the latter organelle in adhesion is unknown.

Strains of $E$. coli that attach intimately and efface microvilli from intestinal epithelial cells have been named 'attaching-effacing E. coli' (AEEC) (Moon et al., 1983) and it has been shown that attachingand-effacing activity occurs in EPEC strains belonging to classes I and II (Moon et al., 1983, Tzipori et al., 1985). Thus, that adhesion would appear to be a general property of EPEC strains and to be independent of both LA and DA.

The situation has been clarified in a recent study by Knutton et al. (1987b) who demonstrated that EPEC adherence is a two-stage mechanism: (i) an initial attachment of bacteria to the intestinal mucosa in a non-intimate fashion is promoted by plasmid-encoded adhesins, e.g., LA and DA; and (ii) effacing of microvilli and intimate EPEC attachment, which may occur in the absence of the first stage, although the plasmid-encoded adhesin enhances the colonising ability of EPEC strains. These workers also presented evidence that the plasmid-encoded LA adhesin involved in the first stage of adhesion is fimbrial in nature (Knutton et al., 1987a). This observation contrasts with those from previous studies (Scotland et al., 1983a; Levine et al., 1985; Andrade and Santa Rosa, 1986b). It was suggested that a small number only of organisms in an EPEC culture are fimbriate which may explain why the fimbriae are difficult to detect. Both fimbriae and outer-membrane proteins may be involved in the first stage of adherence and it is likely that chromosomal genes are involved in the second adhesion stage. The nature of this adhesion, however, and the factors responsible for microvillous disruption are not yet known.

\section{Adherence and pathogenesis}

Although EPEC strains possess toxic and adhesive capabilities which are likely to be involved in the disease process, it has been proposed that the intimate attachment of EPEC strains to intestinal mucosa could disturb the function of the microvillous border and bring about diarrhoea (Moon et al., 1983). Evidence that adhesive non-toxigenic organisms can cause disease has been demonstrated in a distinct class of strains of $E$. coli that show HEp2 adherence but which give negative results in tests for VT, LT and ST. These strains of E. coli do not belong to the EPEC serotypes and are designated enteroadherent E. coli (EAEC) (Mathewson et al., 1985). In epidemiological studies they have been shown to be associated with diarrhoea and their pathogenicity has been confirmed in human volunteers (Mathewson et al., 1986). Diarrhoea caused by EAEC strains is generally less severe than that caused by EPEC strains. As some EPEC strains do not produce detectable Shiga toxin (Marques et al., 1986), they may in fact resemble EAEC strains. It should be noted, furthermore, that HEp2 adhesion in EAEC is distinct from that of EPEC strains (Levine, 1987).

The role of the LA adhesin in disease has been clearly demonstrated. Nevertheless, because strains of EPEC lacking the EAF plasmid can still cause mild diarrhoea in volunteers, it may be that the plasmidless organisms retain some adhesive capacity. The demonstration by Knutton et al. (1987a) that these plasmidless strains are, nevertheless, capable of attaching to epithelia and effacing microvilli is in line with this idea.

Strains of $E$. coli belonging to non-EPEC serogroups but possessing the LA adhesin occur but, on the basis of epidemiological studies, are thought not to be pathogenic; they presumably lack other virulence attributes (Levine, 1987), suggesting that adhesion alone is insufficient to bring about diarrhoea.

\section{Other virulence factors}

Although toxin production and adhesive capabilities are probably the major virulence determinants of EPEC strains, other factors are likely to be involved in helping the organism reach and multiply 
at the site of infection, and in evasion of host defences.

$O$ antigen. It has been demonstrated that both ETEC and EPEC strains have neutral O-antigens but do not have K-polysaccharide capsules, whereas strains belonging to other pathogenic groups of $E$. coli have either acidic O-antigens or charged capsular antigens (Jann and Jann, 1985). With EPEC strains restricted to certain $O$ types, it is possible that the $\mathrm{O}$-antigen has an important, but as yet unidentified, role in EPEC virulence. Possible roles include the ability to take up plasmidmediated virulence factors or evasion of host defences. In support of this is the finding of Sakazaki et al. (1974) that a rough EPEC strain was avirulent in the RIL whereas the smooth parent strain was virulent.

In a strain of $E$. coli that was pathogenic for rabbits and that produces lesions similar to those given by EPEC in vivo, it has been shown that the $\mathrm{O}$-antigen allows bacteria to survive in the intestine by preventing their uptake by the $\mathrm{M}$ cells of Peyers patches (Inman et al., 1986).

Although the $\mathrm{O}$ antigen may be important, it has been shown that within an EPEC O-serogroup only certain $\mathrm{O}: \mathrm{H}$ combinations are enteropathogenic (Wachsmuth, 1980); thus, the O-antigen alone does not specify virulence and other factors must be involved.

Mucinase. Ross (1959) demonstrated mucinase activity in EPEC strains of various serotypes but not in normal-flora strains of $E$. coli. Similar activity has been demonstrated in $V$. cholerae (Finkelstein et al., 1983). The mucinase enzyme of this organism has been shown to be active against lactoferrin, mucin and fibronectin and this ability may contribute to pathogenesis. Further work must be carried out with EPEC strains to detect and characterise mucin-degrading enzymes that might be involved in the breakdown of mucous barriers, thereby allowing bacteria to approach the intestinal membrane.

Aerobactin production. Williams and Roberts (1985) first noted that EPEC strains could produce the iron-chelator aerobactin and suggested that it may be a virulence factor in EPEC strains as it is in other organisms, particularly in those involved in extra intestinal infection. Robins-Browne et al. (1985) could not detect aerobactin production in EPEC strains of proven virulence and argued against its role as a major virulence determinant.

In a later study, aerobactin production was detected in 19 of 51 EPEC strains, whereas none of 19 ETEC strains produced aerobactin (Roberts $e t$ al., 1986); similar unpublished findings have been obtained in this laboratory. There is obviously a major difference in the iron metabolism in these two groups of $E$. coli, related possibly to the avidity of adhesion and the environment in which the organism must grow. EPEC strains adhering intimately to the intestinal mucosa may encounter the iron-binding protein lactoferrin (Roberts et al., 1986).

Thus, although aerobactin production is not essential for EPEC virulence, it may confer some advantages in vivo. Since the production of the Shiga-like toxin is influenced by iron, the ability to take up and utilise this element may be important to EPEC virulence. This is currently under investigation.

Invasive capabilities. Epithelial-cell invasiveness as detected by the ability of the organism to cause keratoconjunctivitis in the guinea-pig eye (Sereny test) is absent in EPEC strains (Goldschmidt and DuPont, 1976; Levine et al., 1978). It has been demonstrated that EPEC adhesion to HEp2 cells is strongly dependent upon bacterial viability and prior cultivation in Ca-containing media. Similar findings are seen with strains of Salmonella and Shigella which, after attachment to animal cells, are internalised (Andrade and Santa Rosa, 1986a). There is evidence that such a process occurs after EPEC adherence to HEp2 cells and intracellular multiplication has been seen to take place (Andrade and Santa Rosa, 1986b). Although invasion is known to occur in tissue culture and in animal tissue (Moon et al., 1983, Knutton et al., 1987b), EPEC invasion of human-intestinal tissue in vivo has not been demonstrated (Rothbaum et al., 1982, 1983).

\section{Conclusion}

Virulence in EPEC strains is clearly multifactorial and there is a great deal yet to be learnt about the relative importance of the various factors discussed. The production of a Shiga-like toxin (with both cytotoxic and enterotoxic properties) is likely to be of major importance and so too is intestinal adhesion.

Adhesion is poorly understood at present and two stages have been identified; there are at least two distinct kinds of adhesion in the first stage (LA and DA). The factors involved in the second stage, and those leading to microvillous disruption, are unknown. It may prove inappropriate to regard EPEC as a homogeneous group of organisms. If different virulence factors are produced by strains of different serotypes, this will necessitate subdivision based on virulence mechanisms. 
Study of virulence factors has important practical implications. For vaccine production, we need to know which determinants are important in the causation of disease so that an effective vaccine may be developed. Diagnosis of disease may be simplified and improved if specific virulence factors can be detected in faecal samples or in strains of $E$. coli isolated from faeces.

Until tests such as DNA probes become commercially available, O-serogrouping of strains of $E$.

\section{REFERENCES}

Andrade J R C, Santa Rosa M R 1986a Attachment and intracellular penetration of classic enteropathogenic Escherichia coli into Hep-2 cells. Revista de Microbiologia, Sao Paulo 17: 53-57.

Andrade J R C, Santa Rosa M R $1986 b$ Investigation on an adhesive property (localised adherence) characteristic of classical enteropathogenic serotypes of Escherichia coli. Revista de Microbiologia, Sao Paulo 17: 116-125.

Baldini M M, Kaper J B, Levine M M, Candy D C A, Moon H W 1983 Plasmid-mediated adhesion in enteropathogenic Escherichia coli. Journal of Pediatric Gastroenterology and Nutrition 2: 534-538.

Bray J 1945 Isolation of antigenically homogeneous strains of Bact. coli neopolitanum from summer diarrhoea of infants. Journal of Pathology and Bacteriology 57 : 239-247.

Charney A N, Gots R E, Formal S B, Gianella R A 1976 Activation of intestinal mucosal adenylate cyclase by Shigella dysenteriae 1 enterotoxin. Gastroenterology 70: 1085-1090.

Cleary T G, Mathewson J J, Faris E, Pickering L K 1985 Shigalike cytotoxin production by enteropathogenic Escherichia coli serogroups. Infection and Immunity 47: 335-337.

Cravioto A, Gross R J, Scotland S M, Rowe B 1979 An adhesive factor found in strains of Escherichia coli belonging to the traditional infantile enteropathogenic serotypes. Current Microbiology 3: 95-99.

De S N, Bhattacharya K, Sarkar J K 1956 A study of the pathogenicity of strains of Bacterium coli from acute and chronic enteritis. Journal of Pathology and Bacteriology 71: 201-209.

De S N, Chatterjee D N 1953 An experimental study of the mechanism of action of Vibrio cholerae on the intestinal mucous membrane. Journal of Pathology and Bacteriology 66: $559-562$.

Donowitz M, Keusch G T, Binder H J 1975 Effect of shigella enterotoxin on electrolyte transport in rabbit ileum. Gastroenterology 69: 1230-1237.

Evans D G, Evans D J, Gorbach S L 1973 Identification of enterotoxigenic Escherichia coli and serum antitoxin activity by the vascular permeability factor assay. Infection and Immunity 8: 731-735.

Evans D J, Evans D G, DuPont H L 1979 Hemagglutination patterns of enterotoxigenic and enteropathogenic Escherichia coli determined with human, bovine, chicken and guinea pig erythrocytes in the presence and absence of mannose. Infection and Immunity 23: 336-346.

Finkelstein R A, Boesman-Finkelstein M, Holt P 1983 Vibrio cholerae hemagglutinin/lectin/protease hydrolyzes fibronectin and ovumucin. F. M. Burnet revisited. Proceedings coli remains the only method for routine detection of EPEC strains in the clinical microbiology laboratory. Positive reactions must be confirmed by tube agglutination with specific antisera and, where possible, by $\mathrm{H}$-agglutination tests.

I should like to thank Drs H. R. Ingham and K. M. Wilkie and my colleagues in the Microbiology Department of Freeman Hospital for advice and constructive criticism during the preparation of this paper.

of the National Academy of Sciences of the USA. 80: 10921095.

Flores J, Grady G F, McIver J, Witkum P, Beckmann B, Sharp G W G 1974 Comparison of the effects of enterotoxins of Shigella dysenteriae and Vibrio cholerae on the adenylate cyclase system of the rabbit intestine. Journal of Infectious Diseases 130: 374-379.

Froman G, Switalski L M, Faris A, Wadstrom T, Hook M 1984 Escherichia coli binding to fibronectin: a mechanism of tissue adherence. Federation Proceedings 43, abstract no. $554, \mathrm{p} 379$.

Goldschmidt M C and DuPont H L 1976 Enteropathogenic Escherichia coli: lack of correlation of serotype with pathogenicity. Journal of Infectious Diseases 133: 153-156.

Gorbach S L, Khurana C M 1972 Toxigenic Escherichia coli a cause of infantile diarrhea in Chicago. New England Journal of Medicine 287: 791-795.

Gross R J, Scotland S M, Rowe B 1976 Enterotoxin testing of Escherichia coli causing epidemic infantile enteritis in the UK. Lancet 1 : 629-631.

Guerrant R L, Brunton L L, Schnaitman T C, Rebhun L I, Gilman A G 1974 Cyclic adenosine monophosphate and alteration of Chinese Hamster ovary cell morphology: a rapid sensitive in vitro assay for the enterotoxins of Vibrio cholerae and Escherichia coli. Infection and Immunity 10: 320-327.

Inman L R, Cantey J R, Formal S B 1986 Colonization, virulence and mucosal interaction of an enteropathogenic Escherichia coli (strain RDEC-1) expressing shigella somatic antigen in the rabbit intestine. Journal of Infectious Diseases 154: 742-751.

Jann K, Jann B 1985 Cell surface components and virulence: Escherichia coli $\mathrm{O}$ and $\mathrm{K}$ antigens in relation to virulence and pathogenicity. In: Sussman M (ed) The virulence of Escherichia coli. Academic Press (for Society for General Microbiology) London, pp 157-176.

Johnson W M, Lior H, Bezanson G S 1983 Cytotoxic Escherichia coli $0157: \mathrm{H} 7$ associated with haemorrhagic colitis in Canada. Lancet 1: 76.

Kantor H S, Tao P, Gorbach S L 1974 Stimulation of intestinal adenyl cyclase by Escherichia coli enterotoxin. Comparison of strains from an infant and an adult with diarrhea. Journal of Infectious Diseases 129 : 1-9.

Karch H, Heesemann J, Laufs R 1987 Phage-associated cytotoxin production by and enteroadhesiveness of enteropathogenic Escherichia coli isolated from infants with diarrhoea in West Germany. Journal of Infectious Diseases 155: 707-715.

Karmali M A, Petric M, Lim C, Fleming P C, Steele B T $1983 a$ Escherichia coli cytotoxin, haemolytic-uraemic syndrome and haemorrhagic colitis. Lancet 2: 1299-1300.

Karmali M A, Steele B T, Petric M, Lim C $1983 b$ Sporadic cases 
of haemolytic-uraemic syndrome associated with faecal cytotoxin and cytotoxin producing Escherichia coli in stools. Lancet 1 : 619-620.

Keusch G T, Donohue-Rolfe A, Jacewicz M 1981 Shigella toxin(s): description and role in diarrhoea and dysentery. Pharmacology and Therapeutics 15 : 403-438.

Klipstein F A, Rowe B, Engert R F, Short H B, Gross R J 1978 Enterotoxigenicity of enteropathogenic serotypes of Escherichia coli isolated from infants with epidemic diarrhea. Infection and Immunity 21 : 171-178.

Knutton S, Baldini M M, Kaper J B, McNeish A S $1987 a$ Role of plasmid-encoded adherence factors in adhesion of enteropathogenic Escherichia coli to HEp-2 cells. Infection and Immunity 55: 78-85.

Knutton S, Lloyd D R, McNeish A S $1987 b$ Adhesion of enteropathogenic Escherichia coli to human intestinal enterocytes and cultured human intestinal mucosa, Infection and Immunity 55: 69-77.

Konowalchuk J, Speirs J I, Stavric S 1977 Vero response to a cytotoxin of Escherichia coli. Infection and Immunity 18: 775-779.

Konowalchuk J, Dickie N, Stavric S, Speirs J I 1978 Properties of an Escherichia coli cytotoxin. Infection and Immunity 20: 575-577.

Law D, Wilkie K M, Freeman R 1987 Examination of enteropathogenic Escherichia coli for an adenylcyclase stimulating factor. Journal of Medical Microbiology 23: 335338.

Levine M M 1987 Escherichia coli that cause diarrhoea: enterotoxigenic, enteropathogenic, enteroinvasive, enterohemorrhagic, and enteroadherent. Journal of Infectious Diseases 155: 377-389.

Levine M M, Edelman R 1984 Enteropathogenic Escherichia coli of classic serotypes associated with infant diarrhea: epidemiology and pathogenesis. Epidemiologic Review 6: 31-51.

Levine M M et al. 1978 Escherichia coli strains that cause diarrhoea but do not produce heat-labile or heat-stable anterotoxins and are non-invasive. Lancet 1 : 1119-1122.

Levine M M et al. 1985 The diarrhoeal response of humans to some classic serotypes of enteropathogenic Escherichia coli is dependent on a plasmid encoding an entero-adhesiveness factor. Journal of Infectious Diseases 152: 550-559.

Long-Krug S A, Weikel C S, Tiemens K T, Hewlett E L, Levine M M, Guerrant R L 1984 Does enteropathogenic Escherichia coli produce heat-labile enterotoxin, heat stable enterotoxins $\mathrm{a}$ or $\mathrm{b}$, or cholera toxin A subunits? Infection and Immunity 46: $612-614$.

Marques L R M, Moore M A, Wells J G, Wachsmuth I K, O'Brien A D 1986 Production of Shiga-like toxin by Escherichia coli. Journal of Infectious Diseases 154 : 338-341.

Mathewson J J et al. 1985 A newly recognized cause of travelers diarrhea : enteroadherent Escherichia coli. Journal of Infectious Diseases 151 : 471-475.

Mathewson J J, Johnson P C, DuPont H L, Satterwhite T K, Winsor D K 1986 Pathogenicity of enteroadherent Echerichia coli in adult volunteers. Journal of Infectious Diseases 154: 524-527.

Moon H W, Whipp S C, Argenzio R A, Levine M M, Gianella R A 1983 Attaching and effacing activities of rabbit and human enteropathogenic Escherichia coli in pig and rabbit intestines. Infection and Immunity 41 : 1340-1351.

Nataro J P, Baldini M M, Kaper J B, Black R E, Bravo N, Levine M M $1985 a$ Detection of an adherence factor of enteropathogenic Escherichia coli with a DNA probe. Journal of Infectious Diseases 152: 560-565.

Nataro J P, Scaletsky I C A, Kaper J B, Levine M M, Trabulsi
L R $1985 b$ Plasmid-mediated factors conferring diffuse and localized adherence of enteropathogenic Escherichia coli. Infection and Immunity 48: 378-383.

Neter E, Westphal O, Luderitz O, Gino R M, Gorzynski E A 1955 Demonstration of antibodies to enteropathogenic Escherichia coli in sera of children of various ages. Paediatrics 16: 801-808.

O'Brien A D, LaVeck G D 1983 Purification and characterization of a Shigella dysenteriae 1-like toxin produced by Escherichia coli. Infection and Immunity 40: 675-683.

O'Brien A D, LaVeck G D, Thompson M R, Formal S B 1982 Production of Shigella dysenteriae type-1 like cytotoxin by Escherichia coli. Journal of Infectious Diseases 146: 763-769.

O'Brien A D, Lively T A, Chen M E, Rothman S W, Formal S B 1983 Escherichia coli O157:H7 strains associated with haemorrhagic colitis in the United States produce a Shigella dysenteriae 1 (shiga) like cytotoxin. Lancet $1: 702$.

O'Brien A D, Chen M E, Holmes R K, Kaper J, Levine M M $1984 a$ Environmental and human isolates of Vibrio cholerae and Vibrio parahaemolyticus produce a Shigella dysenteriae 1 (shiga)-like cytotoxin. Lancet $1: 77-78$.

O'Brien A D, Newland J W, Miller S F, Holmes R K, Smith H W, Formal S B $1984 b$ Shiga-like toxin-converting phages from Escherichia coli strains that cause hemorrhagic colitis or infantile diarrhoea. Science 226: 694-696.

Polotsky et al. 1977 Pathogenic effect of enterotoxigenic Escherichia coli and Escherichia coli causing infantile diarrhoea. Acta Microbiologica Hungarica 24: 221-236.

Punyashthiti K, Finkelstein R A 1971 Enteropathogenicity of Escherichia coli. I Evaluation of mouse intestinal loops. Infection and Immunity 4: 473-478.

Riley L W et al. 1983 Hemorrhagic colitis associated with a rare Escherichia coli serotype. New England Journal of Medicine 308: 681-685.

Roberts M, ParthaSarathy S, Lam-Po-Tang M K L, Williams P $\mathrm{H} 1986$ The aerobactin iron uptake system in enteropathogenic Escherichia coli: evidence for an extinct transposon. FEMS Microbiology Letters 37: 215-219.

Robins-Browne R M, Levine M M, Rowe B, Gabriel E M 1982 Failure to detect conventional enterotoxins in classical enteropathogenic (serotyped) Escherichia coli strains of proven pathogenicity. Infection and Immunity 38: 798-801.

Robins-Browne R M, Feain R, Stuart S J 1985 Aerobactinmediated uptake of iron is not an essential virulence determinant of enteropathogenic Escherichia coli. Lancet 2: 164.

Ross C A C 1959 Mucinase activity of intestinal organisms. Journal of Pathology and Bacteriology 77: 642-644.

Rothbaum R, McAdams A J, Gianella R, Partin J C 1982 A clinicopathologic study of enterocyte-adherent Escherichia coli: a cause of protracted diarrhoea in infants. Gastroenterology 83 : 441-454.

Rothbaum R J, Partin J C, Saalfield K, McAdams A J 1983 An ultrastructural study of enteropathogenic Escherichia coli infection in human infants. Ultrastructural Pathology 4 : 291-304.

Rowe B 1979 The role of Escherichia coli in gastroenteritis. Clinics in Gastroenterology 8: 625-644.

Rowe B, Gross R J, Scotland S M 1975 Serotyping of enteropathogenic Escherichia coli. Lancet 2: 925-926.

Sack R B 1976 Serotyping of E. coli. Lancet 1: 1132.

Sakazaki R, Tamura K, Nakamura A, Kurata T, Gohda A, Takeuchi S 1974 Enteropathogenicity and enterotoxigenicity of human enteropathogenic Escherichia coli. Japanese Journal of Medical Science and Biology 27: 19-33.

Scaletsky I C A, Silva M L M, Trabulsi L R 1984 Distinctive 
patterns of adherence of enteropathogenic Escherichia coli to HeLa cells. Infection and Immunity 45: 534-536.

Scheftel J M, Martin C, Bober C, Monteil H 1980 Isolation of an enterotoxic factor elaborated by human enteropathogenic Escherichia coli. FEMS Microbiology Letters 9: 125130.

Scotland S M, Day N P, Rowe B 1980 Production of a cytotoxin affecting Vero cells by strains of Escherichia coli belonging to traditional enteropathogenic serogroups. FEMS Microbiology Letters 7: 15-17.

Scotland S M, Richmond J E, Rowe B 1983a Adhesion of enteropathogenic strains of Escherichia coli(EPEC) to HEp2 cells is not dependent on the presence of fimbriae. FEMS Microbiology Letters 20 : 191-195.

Scotland S M, Smith H R, Willshaw G A, Rowe B $1983 b$ Vero cytotoxin production in a strain of Escherichia coli is determined by genes carried on bacteriophage. Lancet 2 : 216.

Scotland S M, Smith H R, Rowe B 1985 Two distinct toxins active on Vero cells from Escherichia coli 0157. Lancet 2: 885-886.

Sherman P M, Houston W L, Boedeker E C 1985 Functional heterogeneity of intestinal Escherichia coli strains expressing type 1 somatic pili (fimbriae): assessment of bacterial adherence to intestinal membranes and surface hydrophobicity. Infection and Immunity 49: 797-804.

Smith H R, Day N P, Scotland S M, Gross R J, Rowe B 1984 Phage-determined production of vero cytotoxin in strains of Escherichia coli serogroup 0157. Lancet 1: 1242-1243.

Staley T E, Jones E W, Corley L D 1969 Attachment and penetration of Escherichia coli into intestinal epithelium of the ileum in newborn pigs. American Journal of Pathology 56: 371-392.

Strockbine N A, Marques L R M, Newland J W, Smith H W, Holmes R K, O'Brien A D 1986 Two toxin-converting phages from Escherichia coli O157:H7 strain 933 encode antigenically distinct toxins with similar biologic activities. Infection and Immunity 53: 135-140.

Taylor J, Maltby M P, Payne J M 1958 Factors influencing the response of ligated rabbit-gut segments to injected Escherichia coli. Journal of Pathology and Bacteriology 76: 491499.

Taylor J, Wilkins M P, Payne J M 1961 Relation of rabbit gut reaction to enteropathogenic Escherichia coli. British Journal of Experimental Pathology 42: 43-48.

Tzipori S, Robins-Browne R M, Gonis G, Hayes J, Withers M, McCartney E 1985 Enteropathogenic Escherichia coli enteritis: evaluation of the gnotobiotic piglet as a model of human infection. Gut 26: 570-578.

Ulshen M H, Rollo J L 1980 Pathogenesis of Escherichia coli gastroenteritis in man-another mechanism. New England Journal of Medicine 302: 99-101.

Wachsmuth I K 1980 Escherichia coli: typing and pathogenicity. Clinical Microbiology Newsletter 2(12): 4-7.

Wade W G, Thom B T, Evans N 1979 Cytotoxic enteropathogenic Escherichia coli. Lancet 2: 1235-1236.

Wadstrom T, Baloda S B, Bettelheim K A, Steinruck H, Froman G 1986 Fibronectin binding to enterotoxigenic, enteropathogenic, enteroinvasive (ETEC, EPEC, EIEC) and uropathogenic Escherichia coli. A preliminary report. Abstracts of XIV International Congress of Microbiology 1986: 267.

Williams P H, Roberts M 1985 Aerobactin-mediated iron uptake: a virulence determinant in enteropathogenic Escherichia coli. Lancet 1: 763.

Zinkernagel R M, Colombini A 1975 Passive oral immunization with bovine immunoglobulins: enteropathogenic Escherichia coli from infants and bovine anti-E. coli lactoserum assayed in the rabbit ileal loop model. Medical Microbiology and Immunology 162 : 1-7. 